
\title{
28 Research Suare \\ Iron Therapy-Induced Leukopenia in Arab Female Population, an experience in Qatar
}

\author{
Hussam Almasri ( $\nabla$ hussamalmasri10@gmail.com ) \\ Hamad Medical Corporation https://orcid.org/0000-0002-9549-8352 \\ Ashraf Soliman \\ Hamad Medical Corporation \\ Vincenzo Desanctis \\ University of Ferrara: Universita degli Studi di Ferrara \\ Arwa Alsaud \\ Hamad Medical Corporation
}

Roua Alhashimy

Hamad Medical Corporation

Mona Babikr

Hamad Medical Corporation

Rita Ahmad

Hamad Medical Corporation

Majd Aldwairi

Hamad Medical Corporation

Mohammad Kloub

Hamad Medical Corporation

Shaima Ahmed

Hamad Medical Corporation

Mustafa Al-Tikrity

Hamad Medical Corporation

Mahmoud Eisa

Hamad Medical Corporation

Mohamed Yassin

Hamad Medical Corporation

\section{Research Article}

Keywords: leukopenia, iron therapy, iron deficiency anemia

Posted Date: May 25th, 2021 
DOI: https://doi.org/10.21203/rs.3.rs-524820/v1

License: (c) (i) This work is licensed under a Creative Commons Attribution 4.0 International License. Read Full License 


\section{Abstract}

Introduction

Iron deficiency anemia (IDA) is the most common cause of anemia in both developed and developing countries. Leukopenia is an infrequent side effect of iron therapy reported in the literature as sporadic cases. We conducted a study to clarify this issue and to check its consequences in a big cohort of patients with IDA.

Objective

To assess the relationship between iron therapy (intravenous) and leukopenia, neutropenia or lymphocytopenia, and its clinical impact, if any, on patients.

Materials and Methods

this is a retrospective study conducted in Hamad Medical Corporation, Doha/Qatar. the clinical and biochemical data of 1567 females with IDA who attended the hematology clinic and were treated with intravenous iron therapy were collected and analysed. Complete and differential blood counts and iron profile were studied before and after iron treatment. In addition, cases who developed infections during the time of leukopenia were noted and checked for possible complications.

Results

After iron therapy, out of 1567 case of iron deficiency anemia, 30 cases (1.914\%) developed leukopenia,15 cases $(0.957 \%)$ developed neutropenia and 12 cases $(0.765 \%)$ developed lymphocytopenia. All had normal WBC counts before treatment. 2 patients $(6.66 \%)$ had infection, 1 had upper respiratory tract infection and 1 urinary tract infection, the latter was treated with antibiotics. There were no reported infections during or after iron therapy.

Conclusions

Leukopenia in form of neutropenia or lymphocytopenia may occur as a side effect of IV iron therapy, however, its clinical significance appeared to be limited.

\section{Introduction}

Iron deficiency (ID) is the most common cause of anemia world-wide, and it accounts for the most common nutritional deficiency. Iron deficiency anemia (IDA) particularly affects females in the childbearing age and children at the pre-school age.

Causes of iron deficiency include malnutrition (mainly in developing countries), parasitic infections like hookworm, schistosomiasis, bleeding from any source, malabsorption syndromes. 
The signs and symptoms can be linked to impaired oxygen delivery to the tissues causing fatigue, exertional dyspnea, light-headedness and pallor, or linked to iron deficiency like pica, ice craving, beeturia and restless leg syndrome. $(1,2)$

Anemia was found to account for $8.8 \%$ of total disability from all conditions in one large study. (1)

IDA has been linked to impaired mental and motor development in children (3), preterm birth, heart failure and may predispose to infection and increase mortality rates in peri-operative settings. $(4,5)$

Early diagnosis of IDA is vital to treat early and avoid complications. Treating the cause of IDA and iron replacement are the mainstay of the management. Intravenous iron replacement is currently in wide use because of the advantage of overcoming gastric intolerance and constipation that occur with oral iron and the advantage of rapid replacement of iron.

Low count of circulating white blood cells (WBC) is the definition of leukopenia which can be at the expense of neutrophils, lymphocytes, or both. Leukopenia comes from various causes and usually needs holistic approach.

Leukopenia, neutropenia or lymphocytopenia; as a side effect of iron therapy, has only been reported as sporadic cases in the literature and its clinical significance has not been studied.

We conducted this study to measure the prevalence of Leukopenia, neutropenia or lymphocytopenia as a side effect of IV iron therapy in a large cohort of females with IDA.

Objective: To assess the relationship between iron therapy (intravenous) and leukopenia, neutropenia or lymphocytopenia, and its impact on patient's clinical settings.

\section{Materials And Methods}

We retrospectively reviewed the electronic medical records of patients attended Haematology clinic for IDA and treated with intravenous iron (ferric carboxymaltose or iron saccharide) over 2 years (1-2017: 1-2019) in Hamad Medical Corporation, Doha, Qatar.

All adult females with IDA who received IV iron were included. Anemia due to other etiologies and conditions (including other medications) that may alter WBCs count were excluded. Age, Ethnicity, BMI, Complete blood count and iron studies data were collected using electronic health records (Cerner) before and after treatment with IV iron therapy. Data about concomitant infection at the time of IDA, during or after IV iron therapy, the use of antibiotics and infection related complications were collected. Leukopenia was defined as WBCs count less than 4000/microlitre, Neutropenia as ANC less than 1500/microlitre and lymphocytopenia as lymphocytes less than 1000/microlitre.

\section{Statistical analysis:}


Data were presented as mean $+/-$ SD. Paired $t$ test was used to compare data for patients before versus after iron therapy when the data was normally distributed and mann whitney $u$ test was used when the data was not normally distributed. Significance was accepted when $p<0.5$.

\section{Results}

After iron therapy, out of 1567 case of iron deficiency anemia, 30 cases (1.914\%) developed leukopenia,15 cases $(0.957 \%)$ developed neutropenia and 12 cases $(0.765 \%)$ had lymphocytopenia. All had normal readings before treatment. (tables 1-3)

Only 2 patients $(6.66 \%)$ had infection during the period of iron therapy. One had upper respiratory tract infection and the other had urinary tract infection. The latter was treated with antibiotics. No other patients had recorded infection.

\section{Discussion}

Leukocytopenia is defined as low count of circulating WBCs. This can be caused by low neutrophils count, low lymphocytes count, other WBCs components or combined. Neutropenia can be primary idiopathic or secondary to many conditions including viral infections, hematological diseases, thyroid disorders, drug related or autoimmune diseases. (6) Lymphopenia has a broad variety of causes, most importantly are viral infections, hematological disorders and corticosteroids therapy, $(7,8)$

Iron deficiency has been proposed to be infrequently associated with neutropenia and lymphopenia when other causes are ruled out. (9-11)

Although parenteral iron replacement has a relatively high safety profile, previous case reports suggested a link between iron therapy and impaired production of cell lineages like thrombocytes and leukocytes. The burden of leukopenia/neutropenia/lymphopenia as a consequent of iron therapy has not been well addressed in the literature. One review of 11 case reports and a case series described that thrombocytopenia can be induced by iron replacement (ferrous sulphate).(12)

Brito-Babapulle et al reported a case of fatal bone marrow suppression linked to ferric carboxymaltose therapy in a patient with IDA. This case started as amegacaryocytic thrombocytopenia and erythroid cell aplasia which was followed by a drop in neutrophils count. Other causes were well excluded death occurred before a donor for bone marrow transplant was found. (13) Another case report described the occurrence of neutropenia in a man after oral iron therapy that was transient and improved 1 month after stopping the iron tablets. (14) In our study the incidence of leukopenia, neutropenia and lymphopenia were with parental iron therapy was $1.9,0.95,0.76$ percent, respectively in a large cohort of females treated with intravenous iron for IDA. 
Iron is required for the oxidative response of neutrophils to allow the production of reactive oxygen species (ROS). However, neutrophil function may be severely altered in conditions of iron overload, as observed in chronically transfused patients. Therefore, a tight regulation of neutrophil iron homeostasis seems to be critical for avoiding iron toxicity.

In animal models, it was found that iron-dependent increase of hepatic hepcidin resulted in neutrophil intracellular iron trapping and consecutive defects in oxidative burst activity. Moreover, systemic iron overload has been correlated with a surprising neutrophil priming and resulted in a more powerful oxidative burst. (15)

Excessive iron may impair haematopoiesis, although the mechanisms of this deleterious effect is not entirely known.

In vitro tests show that commonly available intravenous iron formulations have differing capacities to saturate transferrin directly: Iron gluconate > iron sucrose > iron dextran. Intravenous iron treatment produces oxidative stress, as demonstrated by increases in plasma levels of lipid peroxidation products (malondialdehyde), at a point that is much earlier than the time to peak concentration of catalytically active iron, suggesting a direct effect of iron sucrose on oxidative stress. (16)

In animal models, iron overload was found to have a negative impact on the hematopoietic system through the accumulation of the reactive oxygen species (ROS) and its effect on adhesion molecules and cytokine production. It was suggested that ferrous ammonium sulfate can mediate cell apoptosis and cause growth arrest in immature cells.(17) One study found that ferrous ammonium sulphate (FeAS), induced growth arrest and apoptosis in immature hematopoietic cells, which was mediated via reactive oxygen species (ROS) activation of p38MAPK and JNK pathways. (17)

It has been postulated that i.v. iron might promote infection but there are conflicting studies in the literature. The risk of infection is thought partly to be because of some i.v. irons having a potentially immune activating effect. Iron sucrose can induce phenotypical and functional monocytic alterations and have a higher potential to modulate monocyte differentiation to macrophages and mature dendritic cells than more stable preparations. $(18,19)$

In our cohort, with normal renal function, there was no significant association with infections, with only 2 infections reported in 1567 females who received i.v. iron therapy.

A few small trials in CKD populations suggest an increased infection risk with i.v. iron. Agarwal et al. undertook a single-centre RCT that randomly assigned no dialysis-dependent CKD (NDD-CKD) patients with IDA to either oral iron (69 patients) or i.v. iron sucrose (67 patients). As a secondary outcome measure, they found an increase in serious adverse events (SAEs) because of infections in patients receiving i.v. iron, with infections in the oral iron group occurring 27 times in 11 patients, whereas in the i.v. iron group, they occurred 37 times in 19 patients; the adjusted RR ratio was $2.12(1.24-3.64), P<0.006)$ $(20,21)$ 
A systematic review and meta-analysis of RCT investigated the safety and efficacy of i.v. iron therapy. They obtained data from Medline, Embase, and the Cochrane Central Register of Controlled Trials from 1966 to June 2013. In total, 72 trials with 10605 patients were included. Intravenous iron was found to be associated with a significant increase in RR of infection of 1.33 (95\% Cl: 1.10-1.64) compared with oral or no iron supplementation. (22)

However, these findings were subject to bias as infection was not a predefined endpoint in many of the trials and patients had renal disorders. They could also not detect a dose-response association between iron and risk of infection.(23)

Other studies have shown contrasting results regarding risk of infections associated with i.v. iron. In another meta-analysis that included 103 RCT, Avni et al. concluded that there was no increased risk of infections with the use of i.v. iron. (24)

More prospective studies are needed to address the possibility of increased infection risk in patients receiving i.v. iron therapy.

\section{Conclusions}

Leukopenia in the form of neutropenia or lymphocytopenia may infrequently occur as a side effect of IV iron therapy, However, its clinical significance appeared to be limited.

\section{References}

1. Kassebaum NJ, Jasrasaria R, Naghavi M, Wulf SK, Johns N, Lozano R, et al. A systematic analysis of global anemia burden from 1990 to 2010. Blood. 2014 Jan 30;123(5):615-24.

2. Rector WG. Pica: its frequency and significance in patients with iron-deficiency anemia due to chronic gastrointestinal blood loss. J Gen Intern Med. 1989 Dec;4(6):512-3.

3. Haas JD, Fairchild MW. Summary and conclusions of the International Conference on Iron Deficiency and Behavioral Development, October 10-12, 1988. Am J Clin Nutr. 1989 Sep 1;50(3):703-5.

4. Rasmussen K. Is There a Causal Relationship between Iron Deficiency or Iron-Deficiency Anemia and Weight at Birth, Length of Gestation and Perinatal Mortality? J Nutr. 2001;131(2S-2):590S. -601S; discussion 601S-603S..

5. Dunne JR, Malone D, Tracy JK, Gannon C, Napolitano LM. Perioperative anemia: an independent risk factor for infection, mortality, and resource utilization in surgery. J Surg Res. 2002 Feb;102(2):237-44.

6. Lima CSP, Paula EV, Takahashi T, Saad STO, Lorand-Metze I, Costa FF. Causes of incidental neutropenia in adulthood. Ann Hematol. 2006 Oct 1;85(10):705-9.

7. Castelino DJ, McNair P, Kay TW. Lymphocytopenia in a hospital population-what does it signify? Aust N Z J Med. 1997 Apr;27(2):170-4.

8. Tavakolpour S, Rakhshandehroo T, Wei EX, Rashidian M. Lymphopenia during the COVID-19 infection: What it shows and what can be learned. Immunol Lett. 2020 Sep;225:31-2. 
9. Almasri HA, Soliman AT, Desanctis V, Al-Tikrity MA, Alsaud AE, Ahmad RW, et al. The Prevalence and Clinical Impact of Leukopenia Among Arab Population with Iron Deficiency Anemia an Experience from Qatar. Blood. 2020 Nov 5;136(Supplement 1):16-16.

10. Almasri HA, Soliman AT, Desanctis V, Alsaud AE, Babikir MM, Kloub MN, et al. The Prevalence and Clinical Impact of Neutropenia Among Arab Population with Iron Deficiency Anemia an Experience from Qatar. Blood. 2020 Nov 5;136(Supplement 1):19-20.

11. Almasri HA, Soliman AT, Desanctis V, Ahmad RW, Al-Tikrity MA, Alhashimy R, et al. The Prevalence of Lymphocytopenia Among Arab Population with Iron Deficiency Anemia an Experience from Qatar. Blood. 2020 Nov 5;136(Supplement 1):15-6.

12. Babikir M, Ahmad R, Soliman A, Al-Tikrity M, Yassin MA. Iron-Induced Thrombocytopenia. A MiniReview of the Literature and Suggested Mechanisms. Cureus [Internet]. 2020 Sep 2 [cited 2020 Nov 20];12(9). Available from: https://www.cureus.com/articles/33160-iron-induced-thrombocytopenia-amini-review-of-the-literature-and-suggested-mechanisms.

13. Brito-Babapulle FM. Bone Marrow suppression Due to ferric Carboxymaltose Infusion. First Reported Case. Blood. 2013 Nov 15;122(21):4673-4673.

14. Kloub MN, Yassin MA. Oral Iron Therapy-Induced Neutropenia in Patient with Iron Deficiency Anemia. Case Rep Oncol. 2020;13(2):721-4.

15. Renassia C, Louis S, Cuvellier S, Boussetta N, Deschemin J-C, Borderie D, et al. Neutrophils from hereditary hemochromatosis patients are protected from iron excess and are primed. Blood Adv. 2020 Aug 18;4(16):3853-63.

16. Bishu K, Agarwal R. Acute injury with intravenous iron and concerns regarding long-term safety. Clin $J$ Am Soc Nephrol CJASN. 2006 Sep;1(Suppl 1):19-23.

17. Tanaka H, Espinoza JL, Fujiwara R, Rai S, Morita Y, Ashida T, et al. Excessive Reactive Iron Impairs Hematopoiesis by Affecting Both Immature Hematopoietic Cells and Stromal Cells. Cells. 2019 Mar;8(3):226.

18. Fishbane S. Review of issues relating to iron and infection. Am J Kidney Dis Off J Natl Kidney Found. 1999 Oct;34(4 Suppl 2):47-52.

19. Fell LH, Zawada AM, Rogacev KS, Seiler S, Fliser D, Heine GH. Distinct immunologic effects of different intravenous iron preparations on monocytes. Nephrol Dial Transplant Off Publ Eur Dial Transpl Assoc - Eur Ren Assoc. 2014 Apr;29(4):809-22.

20. Kalra PA, Bhandari S. Safety of intravenous iron use in chronic kidney disease. Curr Opin Nephrol Hypertens. 2016 Nov;25(6):529-35.

21. Agarwal R, Kusek JW, Pappas MK. A randomized trial of intravenous and oral iron in chronic kidney disease. Kidney Int. 2015 Oct;88(4):905-14.

22. Litton E, Xiao J, Ho KM. Safety and efficacy of intravenous iron therapy in reducing requirement for allogeneic blood transfusion: systematic review and meta-analysis of randomised clinical trials. BMJ. 2013 Aug;15:347:f4822. 
23. Anker SD, Comin Colet J, Filippatos G, Willenheimer R, Dickstein K, Drexler H, et al. Ferric carboxymaltose in patients with heart failure and iron deficiency. N Engl J Med. 2009 Dec;17(25):2436-48. 361.

24. Avni T, Bieber A, Grossman A, Green H, Leibovici L, Gafter-Gvili A. The safety of intravenous iron preparations: systematic review and meta-analysis. Mayo Clin Proc. 2015 Jan;90(1):12-23.

\section{Abbreviations}

Iron Deficiency Anemia (IDA), White Blood Cells (WBCs), Complete Blood Count (CBC), Body Mass Index (BMI), Absolute Neutrophil Count (ANC), Intravenous (IV), Standard Deviation (SD).

\section{Declarations}

\section{Ethics approval}

Ethical approval for the study was obtained from the institutional review board (IRB) at the Medical Research Center (MRC), Hamad Medical Corporation, Doha, Qatar (MRC-01-20-142).

\section{Availability of data and material}

All data generated during this study are included in this published article.

\section{Competing interests}

The authors declare that they have no competing interests.

\section{Funding}

This study funded by Hamad Medical Corporation, Qatar.

The publication of this article was funded by the Qatar National Library.

\section{Authors' contributions}

Mohamed A Yassin : Conceptualization 
Hussam Almasri, Ashraf Tawfik Soliman, Vincenzo Desanctis, Arwa E Alsaud, Ruoa Alhashimy, Mona M Babikir, Rita Ahmad, Majd M Aldwairi, Mohammad N Kloub, Shaima Ahmed, Mustafa A Al-Tikrity, Mahmoud S Eisa: Research design, Data collection, Literature search, Manuscript preparation.

ATS: Statistical analysis, Manuscript preparation

All authors read and approved the final manuscript.

\section{Acknowledgments}

We would like to acknowledge the Hematology Department and Internal Medicine Residency

Program at Hamad Medical Corporation (Qatar) for the logistic support provided to conduct the study. The publication of this article was funded by the Qatar National Library.

\section{Tables}

Table 1: Iron induced leukopenia

\begin{tabular}{l|ccccccccccc} 
Variables & WBC & Hb & MCV & Platelet & ANC & Lymphocyte & Fe level & Fe Sat & TIBC & Transferrin & feritin \\
\hline Before Fe therapy & & & & & & & & & & & \\
mean & $4.7 *$ & 8.85 & 67.9 & 304.27 & $2.5^{*}$ & 1.57 & 10.41 & 19.69 & $70.8^{*}$ & $13.5^{*}$ & 22.00 \\
SD & 0.78 & 1.59 & 10.1 & 63.88 & 0.9 & 0.42 & 17.57 & 26.93 & 22.2 & 30.84 & 65.26 \\
After Fe therapy & & & & & & & & & & & \\
Mean & 3.59 & $11.4^{*}$ & $79 *$ & $257.6^{*}$ & 1.67 & 1.44 & 12.00 & $32.7 *$ & 45.1 & 6.89 & $159^{*}$ \\
SD & 0.24 & 1.46 & 7.57 & 66.61 & 0.47 & 0.31 & 3.91 & 20.22 & 21.8 & 8.94 & 70.00
\end{tabular}

$* \mathrm{p}<0.05$

Table 2: Iron induced neutropenia 


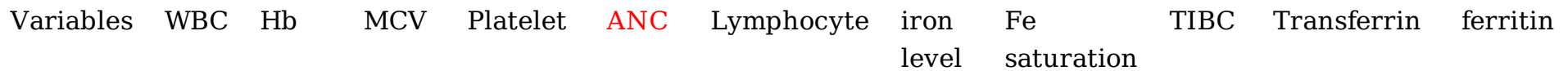

\begin{tabular}{|c|c|c|c|c|c|c|c|c|c|c|c|}
\hline \multirow{2}{*}{$\begin{array}{l}\text { Units } \\
\text { Before } \\
\text { Fe } \\
\text { therapy }\end{array}$} & & & & & & & & & & & \\
\hline & & & & & & & & & & & \\
\hline \multirow{5}{*}{$\begin{array}{l}\text { After Fe } \\
\text { therapy }\end{array}$} & $5.1^{*}$ & 9.5 & 69 & $339 *$ & $2.44^{*}$ & 1.90 & 7.22 & 16.18 & $76.5^{*}$ & 3.21 & 20.3 \\
\hline & 1.37 & 1.3 & 12 & 98.7 & 1.15 & 0.45 & 7.07 & 20.46 & 9.06 & 0.53 & 38.7 \\
\hline & & & & & & & & & & & \\
\hline & 3.75 & $11.8^{*}$ & $81.9 *$ & 295.9 & 1.2 & 1.94 & $14.9 *$ & $29.56 *$ & 58.7 & $6.01 *$ & $126.8^{*}$ \\
\hline & 0.53 & 1.01 & 8.71 & 66.2 & 0.2 & 0.52 & 5.88 & 12.65 & 9.8 & 8.96 & 47.6 \\
\hline
\end{tabular}

$* \mathrm{p}<0.05$

Table 3: Iron induced lymphocytopenia

\begin{tabular}{l|cccccc}
\hline Variables & WBC & Hb & MCV & Platelet & ANC & Lymphocyte \\
\cline { 2 - 7 } $\begin{array}{l}\text { Units } \\
\text { Before Fe therapy }\end{array}$ & & & & & & \\
\cline { 2 - 7 } $\begin{array}{l}\text { Mean } \\
\text { SD }\end{array}$ & 5.25 & 9.48 & 74.31 & $310.4^{*}$ & 3.05 & $1.7^{*}$ \\
\cline { 2 - 7 } After Fe therapy & 1.28 & 1.85 & 8.35 & 90.37 & 1.17 & 0.41 \\
\cline { 2 - 7 } Mean & 7.22 & $11.5^{*}$ & $80.2^{*}$ & 223.75 & $6.14^{*}$ & 0.65 \\
SD & 4.08 & 1.50 & 5.77 & 61.41 & 3.93 & 0.19
\end{tabular}

$* \mathrm{p}<0.05$ 